\title{
Morgan-Voyce Polynomial Approach for Quaternionic Space Curves of Constant Width
}

\author{
Tuba Ağirman Aydin ${ }^{1 *}$, Rabil Ayazoğlu², Hüseyin Kocayiğit ${ }^{3}$
}

\begin{abstract}
The curves of constant width are special curves used in engineering, architecture and technology. In the literature, these curves are considered according to different roofs in different spaces and some integral characterizations of these curves are obtained. However, in order to examine the geometric properties of curves of constant width, more than characterization is required. In this study, firstly differential equations characterizing quaternionic space curves of constant width are obtained. Then, the approximate solutions of the differential equations obtained are calculated by the MorganVoyce polynomial approach. The geometric properties of this curve type are examined with the help of these solutions.
\end{abstract}

Keywords: curve of constant width, quaternionic space curve, Morgan-Voyce polynomial approach

\section{Introduction and preliminary information}

A smooth compact convex plane curve is said to have constant width if the distance between every pair of parallel tangent lines is constant. This constant is called the width of the curve. The simplest example is a circle. Euler was the first to study mathematical properties of noncircular curves of constant width, which he called orbiforms. He proved that the evolvent of a triangular curve is a curve of constant width, and then used the curve of constant width to construct a catoptrix. However, this triangular curve was first explicitly defined by Reuleaux and later named as the Reuleaux triangle. It was proved by Lebesgue and Blaschke that the Reuleaux curved triangle has the smallest area among the curves of a given constant width and that the circle has the largest area [12].

1,2 Faculty of Education, Bayburt University, Bayburt, Turkey;

3 Department of Mathematics, Celal Bayar University, Manisa, Turkey

1* corresponding author: tubaaydin@bayburt.edu.tr ORCID ID: 0000-0001-8034-0723 


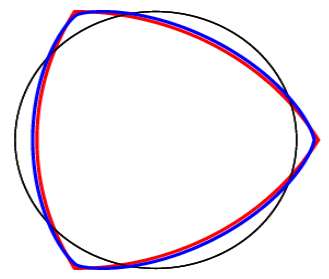

Figure 1. The same width Reuleaux triangle and a circle

The curves of constant width don't pass through their own surface area. They have interesting applications in daily life due to their many different properties like this. The manhole covers are round because a cover in the shape of a disk can't fall through the hole. They can be used as profiles of cams that convert rotary motion into linear motion. It is obviously the Reuleaux curved triangle would have an additional advantage as a coin because, having the least area of all curves of the same constant width, it requires the least material. There are different applications in engineering such as square hole drilling drills and pistons of rotary, internal combustion engines. Water wheels are built on the system that contains this type of curve. The basic curves used in the localization and coverage areas of wireless networks are of this type [17]. There are many decorative applications in art where constant width curve figures play an important role. Especially the most widely known shape of constant width, the circle or circular cross-section cylinder is used in many mechanisms, architecture and design. In the world of the future, different versions of this curve type, other than circle and Reuleaux polygons, can be used in many mechanisms.

The concept of constant width is considered according to different roofs in different spaces and some integral characterizations of these curves are obtained $[1,7,9]$. On the other hand, there are many studies on quaternionic curves $[3,11,14]$. In this section, the concepts related to these two basic structures are discussed.

Irish mathematician Hamilton defines the concept of "quaternion" as a result of his studies to find the equivalent of complex numbers in space. Hamilton discovers these 4-component vectors, called quaternions, with the help of three imaginary units whose square equals -1 . In his later work, he defines the sum and product of quaternions but can't develop a method for division [5].

\subsection{Definition}

In the set of real quaternions $Q_{R}=\left\{q=a_{0} e_{0}+a_{1} e_{1}+a_{2} e_{2}+a_{3} e_{3}\right\}$ the product of the units $\left\{e_{0}, e_{1}, e_{2}, e_{3}\right\}$ as follows:

$$
\begin{aligned}
& e_{0}=1, e_{0}^{2}=1, e_{1}^{2}=e_{2}^{2}=e_{3}^{2}=-1, \\
& e_{1} \cdot e_{2}=e_{3}, e_{2} \cdot e_{3}=e_{1}, e_{3} \cdot e_{1}=e_{2}, \\
& e_{2} \cdot e_{1}=-e_{3}, e_{3} \cdot e_{2}=-e_{1}, e_{1} \cdot e_{3}=-e_{2} .
\end{aligned}
$$


Each element of $Q_{R}$ is called real quaternion and the real numbers $a_{0}, a_{1}, a_{2}, a_{3}$ are called the compenents of $q$. The units $e_{1}, e_{2}, e_{3}$ are taken as the orthogonal coordinate system of the 3-dimensional real vector space. The real quaternion $q$ is expressed as $q=S_{q}+V_{q}$, with the scalar part $S_{q}=a_{0}$ and the vectorial part $V_{q}=a_{1} e_{1}+a_{2} e_{2}+a_{3} e_{3}$ [3]. If there is an equality $q+\alpha q=0$ in the quaternion $q \in Q_{R}$, then $q$ is called a spatial quaternion. It is clear that in order to achieve this equality, the scalar part in the quaternion $q$ must be zero and the vector part must be different from zero. Also, the set of spatial quaternions is isomorphic to the 3 -dimensional vector space. The differentiable curve $\xi$, defined in the form of $\xi: I \subset \mathbb{R} \rightarrow Q_{R} ; s \rightarrow \xi(s)=\sum_{i=1}^{n} \xi_{i}(s) \boldsymbol{e}_{i}$ for $s \in I=[0,1]$, is called a spatial quaternionic curve, in the set of real quaternions $Q_{R}[6]$.

\subsection{Theorem}

Let the spatial quaternionic curve $\xi: I \rightarrow E^{3}$ be given by the arc parameter $s \in[0,1]$. Let be the curvatures $\kappa(s), r(s)$ and the Frenet roof $t(s), n_{1}(s), n_{2}(s)$ at the point $\xi(s)$ of the curve $\xi$. The relationship between the derivatives of vectors and curvatures along the curve is

$$
\begin{aligned}
& t^{\prime}(s)=\kappa(s) n_{1}(s), \\
& n_{1}(s)=\kappa(s) t(s) \mid r(s) n_{2}(s), \\
& n_{2}^{\prime}(s)=-r(s) n_{1}(s) .
\end{aligned}
$$

and the matrix expression is as follows $[2,8]$ :

$$
\left[\begin{array}{l}
t^{\prime} \\
n_{1}^{\prime} \\
n_{2}^{\prime}
\end{array}\right]=\left[\begin{array}{ccc}
0 & \kappa & 0 \\
-\kappa & 0 & r \\
0 & -r & 0
\end{array}\right]\left[\begin{array}{l}
t \\
n_{1} \\
n_{2}
\end{array}\right] .
$$

\subsection{Theorem}

Let a unit-speed curve $\mathrm{C}$ of class $\mathrm{C}^{4}$ have oppositely directed and parallel tangents $\mathrm{t}$ and $\mathrm{t}^{*}$ at opposite points $\alpha$ and $\alpha^{*}$. If the beam joining the opposite points of the curve is a pair of normals, the curve is constant width; on the contrary, if the curve is a curve of constant width, each normal of the curve is a double normal [13]. 


\subsection{Definition}

If the angle that the tangent at a point $\xi(s)$ of the curve $\mathrm{C}$ makes with a fixed direction is called $\varphi$, then the angle $\Delta \varphi$ between the tangents $\vec{t}(s)$ and $\vec{t}(s+\Delta s)$ at points $\xi(s)$ and $\xi(s+\Delta s)$ of this curve is called the contengenesis angle, and the value $\lim _{\Delta s \rightarrow 0} \frac{\Delta \varphi}{\Delta s}=\frac{\partial \varphi}{\partial s}=\kappa(s)$ is called the first curvature of the curve. Also, $\rho=\frac{1}{\kappa}=\frac{\partial s}{\partial \varphi}$ is called the curvature radius of the curve [4].

\subsection{Definition}

Let's consider the differential equation below:

$$
\sum_{k=0}^{m} P_{k}(s) y^{(k)}(s)=g(s), \quad \mathrm{a} \leq s \leq b .
$$

Obviously this is $\mathrm{m}$. order, linear, variable coefficient differential equation. Also, the functions $P_{k}(s), g(s)$ and $y(s)$ are differentiable functions in the range $\mathrm{a} \leq s \leq b$. The Morgan-Voyce polynomial method is developed to find approximate solutions of this equation under certain initial or boundary conditions. Accordingly, the approximate solution can be expressed with Morgan-Voyce polynomials as follows:

$$
y(s) \cong y_{N}(s)=p_{n}(s)=\sum_{n=0}^{N} a_{n} B_{n}(s), \quad N \geq m .
$$

Here, the $a_{n}$ coefficients are defined as Morgan-Voyce polynomial coefficients that must be found. The basis of this method is based on the reduction of the unknown function $y(s)$ to an algebraic system with Morgan-Voyce coefficient $a_{n}$. For this reduction process, the matrix form of the function $y(s)$ and the collocation points $s_{i}=a+\frac{b-a}{N} i, i=0,1, \ldots, N$ are used. Thus, the problem of finding the approximate solutions of a given differential equation or other functional equations becomes the problem of finding the solution of an algebraic matrix equation.

Also, n. order Morgan-Voyce polynomials are expressed as

$$
B_{n}(\mathrm{~s})=\sum_{j=0}^{n}\left(\begin{array}{c}
n+j+1 \\
n-j
\end{array}\right) s^{j}
$$

or recursively as $B_{n}(s)=(s+2) B_{n-1}(s)-B_{n-2}(s), n \geq 2[10,15,16]$.

In this study, the concept of constant width relative to the quaternionic framework on any quaternionic space curve in the 3-dimensional Euclidean space is examined. As a result, differential equations characterizing the quaternionic space curves of constant width are obtained. Then, approximate solutions of these equations are calculated by Morgan-Voyce polynomial approach and some geometric properties are examined. 


\section{Differential equations characterizing the quaternionic space curves of constant width}

Let $\xi$ quaternionic space curve, $x$ and $x^{*}$ be two opposing points on this curve. A simple closed curve with opposite and parallel tangents at its opposite points is expressed by the following equation:

$$
x^{*}(s)=x(s)+\lambda_{1}(s) \vec{t}(\mathrm{~s})+\lambda_{2}(s) \vec{n}_{1}(\mathrm{~s})+\lambda_{3}(s) \vec{n}_{2}(\mathrm{~s}) .
$$

Here, $\vec{t}, \vec{n}_{1}, \vec{n}_{2}$ represents the unit tangent, first normal and second normal, at any point of the curve, respectively. The functions $\lambda_{1}, \lambda_{2}, \lambda_{3}$ are coefficients that determine the curve $[1]$.

\subsection{Theorem}

If the quaternionic space curve $\xi$ is a curve of constant width, it provides the following differential equation with $r \rho=\vartheta$ :

$$
\vartheta\left(\lambda_{1}\right)^{\prime \prime \prime}-\vartheta^{\prime}\left(\lambda_{1}\right)^{\prime \prime}+\vartheta\left(1+\vartheta^{2}\right)\left(\lambda_{1}\right)^{\prime}-\vartheta^{\prime} \lambda_{1}+\vartheta f^{\prime \prime}(\varphi)-\vartheta^{\prime} f^{\prime}(\varphi)+\vartheta^{3} f(\varphi)=0 .
$$

\subsubsection{Proof}

If the Eq. (2) is differentiated according to the arc length parameter s, the following equation is obtained:

$$
\begin{gathered}
-\frac{\partial s^{*} \vec{t}}{\partial s}=\left(\frac{\partial \lambda_{1}}{\partial s}-\kappa \lambda_{2}+1\right) \vec{t}+\left(\frac{\partial \lambda_{2}}{\partial s}+\kappa \lambda_{1}-r \lambda_{3}\right) \vec{n}_{1}+\left(\frac{\partial \lambda_{3}}{\partial s}+r \lambda_{2}\right) \vec{n}_{2}, \\
\text { for } \frac{\partial x^{*}}{\partial s}=t^{*} \frac{\partial s^{*}}{\partial s} \text { and } t^{*}=-t \text {. If both sides of this equation are multiplied by } \frac{\partial s}{\partial \varphi}=\rho,
\end{gathered}
$$

the following equality is obtained:

$$
-\rho^{*} \vec{t}=\left(\frac{\partial \lambda_{1}}{\partial \varphi}-\lambda_{2}+\rho\right) \vec{t}+\left(\frac{\partial \lambda_{2}}{\partial \varphi}+\lambda_{1}-r \rho \lambda_{3}\right) \overrightarrow{n_{1}}+\left(\frac{\partial \lambda_{3}}{\partial \varphi}+r \rho \lambda_{2}\right) \overrightarrow{n_{2}} .
$$

By means of this equality, the following system is obtained: 


$$
\begin{aligned}
& \frac{\partial \lambda_{1}}{\partial \varphi}=\lambda_{2}-f(\varphi), \\
& \frac{\partial \lambda_{2}}{\partial \varphi}=-\lambda_{1}+r \rho \lambda_{3}, \\
& \frac{\partial \lambda_{3}}{\partial \varphi}=-r \rho \lambda_{2} .
\end{aligned}
$$

with $f(\varphi)=\rho-\rho^{*}$. This is Frenet-like differential equation system. If the differential of the Eq. (4.1) according to $\varphi$ is used in the Eq. (4.2), the following expression is obtained:

$$
\lambda_{3}=\frac{1}{r \rho}\left(\lambda_{1}\right)^{\prime \prime}+\frac{1}{r \rho} \lambda_{1}+\frac{1}{r \rho} f^{\prime}(\varphi) .
$$

If the derivative of this equation is used in the Eq. (4.3), the following differential equation is obtained, with $r \rho=\vartheta$ :

$$
\vartheta\left(\lambda_{1}\right)^{\prime \prime \prime}-\vartheta^{\prime}\left(\lambda_{1}\right)^{\prime \prime}+\vartheta\left(1+\vartheta^{2}\right)\left(\lambda_{1}\right)^{\prime}-\vartheta^{\prime} \lambda_{1}+\vartheta f^{\prime \prime}(\varphi)-\vartheta^{\prime} f^{\prime}(\varphi)+\vartheta^{3} f(\varphi)=0
$$

\section{Thus the proof is completed.}

\subsection{Theorem}

If $\xi$ quaternionic space curve is a curve of constant width, it provides the following differential equation, with $r \rho=\vartheta$ :

$$
\left(\lambda_{2}\right)^{\prime \prime \prime}-\frac{\vartheta^{\prime \prime}}{\vartheta^{\prime}}\left(\lambda_{2}\right)^{\prime \prime}+\left(1+\vartheta^{2}\right)\left(\lambda_{2}\right)^{\prime}+\left[3 \vartheta \vartheta^{\prime}-\frac{\vartheta^{\prime \prime}}{\vartheta^{\prime}}\left(1+\vartheta^{2}\right)\right] \lambda_{2}-f=0 .
$$

This is the characterization of the quaternionic space curve of constant width based on the $\lambda_{2}$ coefficient that determines the curve.

\subsection{Theorem}

If $\xi$ quaternionic space curve is a curve of constant width, it provides the following differential equation, with $r \rho=\vartheta$ :

$$
\frac{1}{\vartheta}\left(\lambda_{3}\right)^{\prime \prime \prime}+2\left(\frac{1}{\vartheta}\right)^{\prime}\left(\lambda_{3}\right)^{\prime \prime}+\left[\left(\frac{1}{\vartheta}\right)^{\prime \prime}+\frac{1}{\vartheta}+\vartheta\right]\left(\lambda_{3}\right)^{\prime}+\vartheta^{\prime} \lambda_{3}+f=0 .
$$

This is the characterization of the quaternionic space curve of constant width based on the coefficient $\lambda_{3}$ that determines the curve. 


\section{Investigation of geometric properties of curve type}

Let $\xi$ represent the quaternionic space curve of constant width. Since the distance between the $x$ and $x^{*}$ opposing points of the $\xi$ is constant, $h\left(x, x^{*}\right)=\left\|x-x^{*}\right\|^{2}=\lambda_{1}^{2}+\lambda_{2}^{2}+\lambda_{3}^{2}$ and $\lambda_{1}^{2}+\lambda_{2}^{2}+\lambda_{3}^{2}=$ constant is written. If this equation is differential with respect to $\varphi$, it is clear that the following equation will be obtained:

$$
\lambda_{1}\left(\lambda_{1}\right)^{\prime}+\lambda_{2}\left(\lambda_{2}\right)^{\prime}+\lambda_{3}\left(\lambda_{3}\right)^{\prime}=0 .
$$

If the Eq. (4.2) and Eq. (4.3) are used in this equation, the following expression is obtained:

$$
\lambda_{1}\left(\left(\lambda_{1}\right)^{\prime}-\lambda_{2}\right)=0
$$

Firstly, in the Eq. (7), if $\left(\lambda_{1}\right)^{\prime}-\lambda_{2}=0$, then $\left(\lambda_{1}\right)^{\prime}=\lambda_{2}, f(\varphi)=0$ is obtained from the Eq. (4.1). So the curvature radii of the simple closed quaternionic curve of constant width are equal at opposite points. For a pair of quaternionic curves of constant width, if $f(\varphi)=0$, one of the curves is equal to translated with the constant vector $\vec{d}=\lambda_{1} \vec{t}+\lambda_{2} \vec{n}_{1}+\lambda_{3} \vec{n}_{2}$ of the other. Also, when $f(\varphi)=0$ is taken, it is clear that the Eq. (3) will be as follows:

$$
\vartheta\left(\lambda_{1}\right)^{\prime \prime}-\vartheta^{\prime}\left(\lambda_{1}\right)^{\prime \prime}+\left(\vartheta+\vartheta^{2}\right)\left(\lambda_{1}\right)^{\prime}-\vartheta^{\prime} \lambda_{1}=0
$$

This is the characterization of the quaternionic space curve of constant width based on the coefficient $\lambda_{1}$ that determines the curve. When $f(\varphi)=0$ is taken in Eq. (5) and Eq. (6), the characterization of the quaternionic space curves of constant width according to coefficients $\lambda_{2}$ and $\lambda_{3}$, respectively, is obtained as follows:

$$
\begin{gathered}
\left(\lambda_{2}\right)^{\prime \prime \prime}-\frac{\vartheta^{\prime \prime}}{\vartheta^{\prime}}\left(\lambda_{2}\right)^{\prime \prime}+\left(1+\vartheta^{2}\right)\left(\lambda_{2}\right)^{\prime}+\left[3 \vartheta \vartheta^{\prime}-\frac{\vartheta^{\prime \prime}}{\vartheta^{\prime}}\left(1+\vartheta^{2}\right)\right] \lambda_{2}=0, \\
\frac{1}{\vartheta}\left(\lambda_{3}\right)^{\prime \prime \prime}+2\left(\frac{1}{\vartheta}\right)^{\prime}\left(\lambda_{3}\right)^{\prime \prime}+\left[\left(\frac{1}{\vartheta}\right)^{\prime \prime}+\frac{1}{\vartheta}+\vartheta\right]\left(\lambda_{3}\right)^{\prime}+\vartheta^{\prime} \lambda_{3}=0 .
\end{gathered}
$$

On the other hand,

(i) Let $\lambda_{1}$ be constant:

$$
\begin{aligned}
& \Rightarrow \lambda_{2}=0 \text { from the Eq. (7), } \\
& \Rightarrow f(\varphi)=0 \text { from the Eq. (4.1), } \\
& \Rightarrow \lambda_{3}=\text { const. from the Eq. (4.3), } \\
& \Rightarrow \frac{\lambda_{1}}{\lambda_{3}}=\frac{r}{\kappa}=\text { const. from the Eq. (4.2). }
\end{aligned}
$$

In this case, the $\xi$ quaternionic space curve can be called "quaternionic helix".

(ii) Let $\lambda_{1}=0$ :

$$
\Rightarrow \lambda_{2}=f(\varphi) \text { from the Eq. (4.1), }
$$




$$
\begin{aligned}
& \Rightarrow\left(\lambda_{2}\right)^{\prime}=r \rho \lambda_{3} \text { from the Eq. (4.2), } \\
& \Rightarrow\left(\lambda_{3}\right)^{\prime}=-r \rho \lambda_{2} \text { from the Eq. (4.3). }
\end{aligned}
$$

In this case, with $\lambda_{2}=f(\varphi)$, system (4) can be expressed as a new system as follows:

$$
\begin{aligned}
& \left(\lambda_{2}\right)^{\prime}=r \rho \lambda_{3}, \\
& \left(\lambda_{3}\right)^{\prime}=-r \rho \lambda_{2} .
\end{aligned}
$$

The Eq. (9.1) is differentiated according to $\varphi$. Then, if the Eq. (9.2) is substituted in the expression obtained, a second-order, variable coefficient, homogeneous, linear differential equation based on $\lambda_{2}$ value is obtained as follows:

$$
(r \rho)\left(\lambda_{2}\right)^{\prime \prime}-(r \rho)^{\prime}\left(\lambda_{2}\right)^{\prime}+(r \rho)^{3} \lambda_{2}=0 .
$$

First, Eq. (10) is converted to equation with constant coefficient by changing $\frac{\partial z}{\partial \varphi}=r \rho$ and hence $z=\int(r \rho) \partial \varphi$ independent variable, for solution. If this variable change is applied to the Eq. (10), the equation is obtained as $\left(\frac{\partial z}{\partial \varphi}\right)^{3}\left(\frac{\partial^{2} \lambda_{2}}{\partial z^{2}}+\lambda_{2}\right)=0$. Since $\frac{\partial^{2} \lambda_{2}}{\partial z^{2}}+\lambda_{2}=0$, $\frac{\partial z}{\partial \varphi} \neq 0$. So the solution of the equation $\left(\lambda_{2}\right) "+\lambda_{2}=0$ is obtained as $\lambda_{2}=A \cos \left[\int(r \rho) \partial \varphi\right]+B \sin \left[\int(r \rho) \partial \varphi\right]$.

Similarly, in the Eq. (9.2) $\lambda_{2}$ is left alone and the obtained equation is differentiated according to $\varphi$. If this differential is then substituted in the Eq. (9.1), a second order, variable coefficient, homogeneous, linear differential equation is obtained according to value of $\lambda_{3}$ as follows:

$$
(r \rho)\left(\lambda_{3}\right)^{\prime \prime}-(r \rho)^{\prime}\left(\lambda_{3}\right)^{\prime}+(r \rho)^{3} \lambda_{3}=0 .
$$

And again, following the same method, Eq. (11) is converted to equation with constant coefficient by changing $\frac{\partial z}{\partial \varphi}=r \rho$ and hence $z=\int(r \rho) \partial \varphi$ independent variable, for solution. If this variable change is applied to the Eq. (11), the following equation is obtained:

$$
\left(\frac{\partial z}{\partial \varphi}\right)^{3}\left(\frac{\partial^{2} \lambda_{3}}{\partial z^{2}}+\lambda_{3}\right)=0
$$

Since $\frac{\partial^{2} \lambda_{3}}{\partial z^{2}}+\lambda_{3}=0, \frac{\partial z}{\partial \varphi} \neq 0$. So the solution of the equation $\left(\lambda_{3}\right) "+\lambda_{3}=0$ is obtained as

$$
\lambda_{3}=C \cos \left[\int(r \rho) \partial \varphi\right]+D \sin \left[\int(r \rho) \partial \varphi\right] .
$$


Finally, if these $\lambda_{2}$ and $\lambda_{3}$ values are written in the Eq. (9.1), the following expression is obtained:

$$
-A \sin \left[\int(r \rho) \partial \varphi\right]+B \cos \left[\int(r \rho) \partial \varphi\right]=\operatorname{Ccos}\left[\int(r \rho) \partial \varphi\right]+\operatorname{Dsin}\left[\int(r \rho) \partial \varphi\right] .
$$

Here $D=-A, C=B$ is found. On the other hand, if these equations are used in the constant vector $\vec{d}=\lambda_{1} \vec{t}+\lambda_{2} \vec{n}_{1}+\lambda_{3} \vec{n}_{2}$, the following expression is obtained:

$$
\|\vec{d}\|=\sqrt{\lambda_{1}^{2}+\lambda_{2}^{2}+\lambda_{3}^{2}}=\sqrt{A^{2}+B^{2}} .
$$

\subsection{Theorem}

Let $\xi$ represent quaternionic space curve. Let the tangents and prime normals of this curve at opposite points be parallel to each other and in opposite directions. The beam joining the opposite points of this curve is a pair of normal of the curve, but if and only if the curve is a quaternionic curve of constant width.

\subsubsection{Proof}

$(\Rightarrow)$ Let the constant vector $\vec{d}=\lambda_{1} \vec{t}+\lambda_{2} \vec{n}_{1}+\lambda_{3} \vec{n}_{2}$ be a double normal of the curve. In this case,

$$
h(\vec{d}, \vec{t})=\lambda_{1}=0 \text { and } h\left(\vec{d}, \vec{t}^{*}\right)=0 .
$$

On the other hand, if the Eq. (9.1) is multiplied by $\lambda_{2}$, the Eq. (9.2) by $\lambda_{3}$, and the obtained equations are summed to the side, the following expression is obtained:

$$
\lambda_{2}\left(\lambda_{2}\right)^{\prime}+\lambda_{3}\left(\lambda_{3}\right)^{\prime}=0 \text {. }
$$

If integration is applied to this equation, $\lambda_{2}^{2}+\lambda_{3}^{2}=$ const. is obtained. Therefore, the quaternionic space curve $\xi$ has a constant width, for $\|\vec{d}\|=$ const. .

Let $(\Leftarrow) \xi$ be a quaternionic space curve of constant width. In this case, the distance between opposing points is constant. So $\|\vec{d}\|^{2}=\lambda_{1}^{2}+\lambda_{2}^{2}+\lambda_{3}^{2}=$ const. If derivative is applied to the equation $\lambda_{1}^{2}+\lambda_{2}^{2}+\lambda_{3}^{2}=$ const. and the equations of the system (4) are written in this expression, the following equality is obtained:

$$
\lambda_{1}\left(\frac{\partial \lambda_{1}}{\partial s}-\lambda_{2} \mathrm{k}\right)=0 \rightarrow \lambda_{1}=0
$$

So the constant vector $\vec{d}$ is in plane and $\xi$ is the double normal of the quaternionic space curve of constant width.

\section{Thus the proof is completed.}




\section{Approximate solution with Morgan-Voyce polynomial approach}

In this section, approximate solutions of the differential Eq. (8) that characterizes the quaternionic space curves of constant width based on the coefficient $\lambda_{1}$ will be obtained by the Morgan-Voyce polynomial approach. Similar solution can also be applied for characterizations linked to the coefficients $\lambda_{2}$ and $\lambda_{3}$. Firstly, the differential Eq. (8) is rearranged as follows:

$$
\sum_{k=0}^{3} P_{k}(s) y^{(k)}(s)=g(s), \quad 0 \leq s \leq 2 \pi,
$$

for $P_{3}(s)=\vartheta, P_{2}(s)=P_{0}(s)=-\vartheta^{\prime}, P_{1}(s)=\vartheta+\vartheta^{2}$ and $g(s)=0$. Suppose that this equation has an approximate solution under initial conditions $y^{(k)}(0)=\lambda_{k},(k=0,1,2)$ in the form of Morgan-Voyce polynomials as

$$
y(s)=\sum_{n=0}^{N} a_{n} B_{n}(s) .
$$

Let $N=3$ for convenience. Here, $P_{k}$ and $g$ functions are known functions and $\lambda_{k}$ is suitable constant, $a_{n},(n=0,1, \ldots, N)$ are unknown coefficients, $B_{n}(s)$ are Morgan-Voyce polynomials. The first four of the Morgan-Voyce polynomials are as follows:

$$
B_{0}(s)=1, B_{1}(s)=s+2, B_{2}(s)=s^{2}+4 s+3, B_{3}(s)=s^{3}+6 s^{2}+10 s+4 .
$$

\subsection{Basic matrix relations}

First of all, the approximate solution $y(s)=\sum_{n=0}^{N} a_{n} B_{n}(s)$ can be converted into matrix form $y(s) \cong y_{N}(s)=B(s) A, \quad$ with $\quad B(s)=\left[\begin{array}{llll}B_{0}(s) & B_{1}(s) & B_{2}(s) & B_{3}(s)\end{array}\right] \quad$ and $A=\left[\begin{array}{llll}a_{0} & a_{1} & a_{2} & a_{3}\end{array}\right]^{T}$, for $\mathrm{N}=3$. On the other hand, there is an matrix relation

$$
B(s)=S(s) R^{T} \text { for } S(s)=\left[\begin{array}{llll}
1 & s & s^{2} & s^{3}
\end{array}\right] \text { and } R=\left[\begin{array}{cccc}
1 & 0 & 0 & 0 \\
2 & 1 & 0 & 0 \\
3 & 4 & 1 & 0 \\
4 & 10 & 6 & 1
\end{array}\right]
$$

by using the definition of polynomial. Also, the derivative matrices $B^{\prime}(s)=S(s) T^{T} R^{T}, B^{(k)}(s)=S(s)\left(T^{T}\right)^{k} R^{T}$ are defined, for 


$$
T=\left[\begin{array}{llll}
0 & 0 & 0 & 0 \\
1 & 0 & 0 & 0 \\
0 & 2 & 0 & 0 \\
0 & 0 & 3 & 0
\end{array}\right] .
$$

$y^{(k)}(s) \cong y_{N}^{(k)}(s)=S(s)\left(T^{T}\right)^{k} R^{T} A$ are obtained with the help of these matrices. Also, the matrix relations of the differential part are obtained in the form $\sum_{k=0}^{3} P_{k} Y^{(k)}=G$ by using standard collocation points $s_{i}=\frac{2 \pi}{3} i, i=0,1,2,3$ in the Eq. (12), in the range of $0 \leq s \leq 2 \pi$, for $N=3$. The matrices

$$
\begin{aligned}
& P_{k}=\operatorname{diag}\left[\begin{array}{llll}
P_{k}(0) & P_{k}\left(\frac{2 \pi}{3}\right) & P_{k}\left(\frac{4 \pi}{3}\right) & P_{k}(2 \pi)
\end{array}\right], \\
& Y^{(k)}=\left[\begin{array}{llll}
y^{(k)}(0) & y^{(k)}\left(\frac{2 \pi}{3}\right) & y^{(k)}\left(\frac{4 \pi}{3}\right) & y^{(k)}(2 \pi)
\end{array}\right]^{T}
\end{aligned}
$$

are obvious and the matrix $W=\sum_{k=0}^{3} P_{k} S\left(T^{T}\right)^{k} R^{T}$ is calculated, for $W A=G$ and the equation is written as the augmented matrix $[W ; G]$.

\subsection{Matrix calculations for initial conditions}

Under the initial conditions given as

$$
y(0)=\mu_{0}, y^{\prime}(0)=\mu_{1}, y^{\prime \prime}(0)=\mu_{2}
$$

the matrix expression of the conditions is calculated as

$$
U_{0}=\left[\begin{array}{llll}
1 & 2 & 3 & 4
\end{array}\right], U_{1}=\left[\begin{array}{llll}
0 & 1 & 4 & 10
\end{array}\right], U_{2}=\left[\begin{array}{llll}
0 & 0 & 2 & 12
\end{array}\right] \text {. }
$$

\subsection{Solution}

If the matrix form of conditions is used in the matrix form $[W ; G]$ the following matrix is obtained:

$$
\left[W^{*} ; G^{*}\right]=\left[\begin{array}{cccccc}
1 & 2 & 3 & 4 & ; & \mu_{0} \\
0 & 1 & 4 & 10 & ; & \mu_{1} \\
0 & 0 & 2 & 12 & ; & \mu_{2} \\
\xi_{1} & \xi_{2} & \xi_{3} & \xi_{4} & ; & 0
\end{array}\right]
$$


Here $\xi_{1}, \xi_{2}, \xi_{3}$ and $\xi_{4}$ are clearly expressed as follows:

$$
\begin{aligned}
\xi_{1}= & P_{0}(2 \pi), \xi_{2}=(2+2 \pi) P_{0}(2 \pi)+P_{1}(2 \pi), \\
\xi_{3}= & \left(3+8 \pi+4 \pi^{2}\right) P_{0}(2 \pi)+(4+4 \pi) P_{1}(2 \pi)+2 P_{2}(2 \pi), \\
\xi_{4}= & \left(3+20 \pi+24 \pi^{2}+8 \pi^{3}\right) P_{0}(2 \pi)+\left(10+24 \pi+12 \pi^{2}\right) P_{1}(2 \pi) \\
& +(12+12 \pi) P_{2}(2 \pi)+6 P_{3}(2 \pi) .
\end{aligned}
$$

In addition, the following equations are obvious from the differential Eq. (8):

$$
P_{0}(2 \pi)=P_{2}(2 \pi)=-\vartheta^{\prime}(2 \pi), P_{1}(2 \pi)=\vartheta(2 \pi)+\vartheta^{2}(2 \pi), P_{2}(2 \pi)=\vartheta(2 \pi) .
$$

Finally, with the help of equality $A=W^{*-1} G^{*}, \mathrm{a}_{\mathrm{n}}$ unknowns are calculated as follows:

$$
\begin{aligned}
& a_{0}=\frac{\left(-6 \xi_{3}+14 \xi_{2}+\xi_{4}\right) \mu_{0}+\left(12 \xi_{3}-14 \xi_{2}-2 \xi_{4}\right) \mu_{1}+\left(-8 \xi_{3}-14 \xi_{2}+2.5 \xi_{4}\right) \mu_{2}}{-14 \xi_{1}-6 \xi_{3}+14 \xi_{2}+\xi_{4}}, \\
& a_{1}=\frac{\left(-14 \xi_{1}\right) \mu_{0}+\left(14 \xi_{1}-6 \xi_{3}+\xi_{4}\right) \mu_{1}+\left(-7 \xi_{1}+5 \xi_{3}-2 \xi_{4}\right) \mu_{2}}{-14 \xi_{1}-6 \xi_{3}+14 \xi_{2}+\xi_{4}} \\
& a_{2}=\frac{\left(6 \xi_{1}\right) \mu_{0}+\left(-12 \xi_{1}+6 \xi_{2}\right) \mu_{1}+\left(8 \xi_{1}-5 \xi_{2}+0.5 \xi_{4}\right) \mu_{2}}{-14 \xi_{1}-6 \xi_{3}+14 \xi_{2}+\xi_{4}} \\
& a_{3}=\frac{\left(-\xi_{1}\right) \mu_{0}+\left(2 \xi_{1}-\xi_{2}\right) \mu_{1}+\left(2 \xi_{2}-2.5 \xi_{1}-0.5 \xi_{3}\right) \mu_{2}}{-14 \xi_{1}-6 \xi_{3}+14 \xi_{2}+\xi_{4}}
\end{aligned}
$$

If these values $a_{n}$ are substituted in the Eq. (13), the coefficient $\lambda_{1}(s)$ determining the curve is obtained as follows:

$$
\lambda_{1}(s)=a_{0}+(s+2) a_{1}+\left(s^{2}+4 s+3\right) a_{2}+\left(s^{3}+6 s^{2}+10 s+4\right) a_{3} .
$$

\section{Conclusion}

In this study, the concept of constant width relative to the quaternionic framework on any quaternionic space curve in the 3-dimensional Euclidean space is examined. The differential equation that characterizes constant-breadth quaternionic space curves is obtained according to coefficient $\lambda_{1}$ determining the curve. The geometrical properties of the curve type are interpreted by making the solutions of the obtained equations in closed form. Then, the approximate solution of the equation that characterizes this curve type based on the coefficient $\lambda_{1}$ is calculated by using the Morgan-Voyce polynomial approach method.

This study can be extended to 4-dimensional or even n-dimensional Euclidean space. Similar work can be done for other coefficient functions, and differential equations that characterize the curve can be obtained according to coefficients $\lambda_{2}$ and $\lambda_{3}$. This approximate solution can also be obtained by using different matrix collocation methods like Hermite, Taylor, Bernstein etc. 


\section{References}

[1] Akdoğan Z., Mağden A., Some characterization of curves of constant-breadth in $\mathrm{E}^{\mathrm{n}}$ space, Turkish Journal of Mathematics, 25, 2001, 433-444.

[2] Demir S., Özdaş K., Serret-Frenet formulas by real quaternions, Süleyman Demirel University, Journal of Science Institute, 9, 2005, 1-7.

[3] Hacısalihoğlu H.H., Motion geometry and quaternions theory, Gazi University, Faculty of Arts and Sciences Publications, Ankara, 1983.

[4] Hacısalihoğlu H.H., Differential geometry I, Ankara university Pres, Ankara, 1998.

[5] Hamilton W.R., Lectures on Quaternions, 1853.

[6] Karadağ M., Sivridağ A.İ., Univariate quaternion-valued functions and tendency lines, Erciyes University Journal of Science Institute, 13, 1997, 23 - 36.

[7] Mağden A., Y1lmaz S., On the curves of constant-breadth in four dimensional Galilean space, International Mathematical Forum, 9, 2014, 1229 - 1236.

[8] O'neil B., Elementary differantial geometry, Academic Pres, Newyork, 1966.

[9] Önder M., Kocayiğit H., Candan E., Differential equations characterizing timelike and spacelike curves of constant-breadth in Minkowski 3-space, Journal of the Korean Mathematical Society, 48, 2011, 849-866.

[10] Özel M., Kürkçü Ö.K., Sezer M., Morgan-Voyce matrix method for generalized functional integro-differential equations of volterra-type, Journal of Science and Arts, 2 , 2019, 295-310.

[11]Öztürk G., Kişi İ., Büyükkütük S., Constant Ratio Quaternionic Curves in Euclidean Spaces, Advances in Applied Clifford Algebras, 27, 2017, 1659-1673.

[12]Resnikoff H.L., On Curves and Surfaces of Constant Width, arXiv: Differential Geometry, 2015, 1-48.

[13] Sezer M., Differential equations characterizing space curves of constant-breadth and a criterion for these curves, Doğa Tr J Math, 1989, 69-78.

[14] Soyfidan T., Parlatıcı H., Güngör M.A., On The Quaternionic Curves According to Parallel Transport Frame, TWMS Journal of Pure and Applied Mathematics, 4, 2013,194-203.

[15] Swamy M.N.S., Properties of the polynomial defined by Morgan-Voyce. The Fibonacci Quarterly, 4, 1966, 73-81.

[16] Türkyılmaz B., Gürbüz B., Sezer M., Morgan-Voyce polynomial approach for solution of high-order linear differential-difference equations with residual error estimation, Düzce University Journal of Science \& Technology, 4, 2016, 252-263.

[17]Zhu C., Zheng C., Shu L., Han G., A survey on coverage and connectivity issues in wireless sensor networks, Journal Network and Computer Applications, 35, 2012, 619632.

Received 25.03.2020, Accepted 24.11.2020 